\title{
Impact of length of hospice on spending and utilization among Medicare beneficiaries with lung cancer
}

\author{
Yamini Kalidindi, $\mathrm{MHA}^{1}$, Jeah Jung, $\mathrm{PhD}^{1}$, Joel Segel, $\mathrm{PhD}^{1}$, Douglas Leslie, $\mathrm{PhD}^{2}$ \\ 1.Department of Health Policy and Administration. Pennsylvania State University \\ 2.Department of Public Health Sciences, College of Medicine, Pennsylvania State University, \\ Hershey
}

\section{Abstract}

Objectives: To estimate differences in spending and utilization between hospice users and nonusers with lung cancer by length of hospice enrollment.

Study Design: Retrospective analysis using 2009-2013 Medicare claims.

Methods: The study sample was a 10\% random sample of Medicare fee-for-service beneficiaries with lung cancer who died between 2010 and 2013. We identified different categories of hospice users (hospice enrollment for 1-7 days, 8-14 days, 15-30 days, 31-60 days, 61 days - 6 months) and non-users. We used propensity score matching to match users in each enrollment category with non-users. The outcomes were: a) total Medicare spending, b) number of hospitalizations, c) number of emergency department (ED) visits, d) number of physician-administered chemotherapy claims, and e) number of radiation therapy sessions. Regression analysis was used to compare outcomes between users and non-users by enrollment period.

Results: Hospice users had significantly lower spending, fewer hospitalizations, and fewer ED visits than non-users across all categories of hospice enrollment. Large savings occurred when patients stayed in hospice for at least one month (\$16,566 for those enrolled 61 days - 6 months; $\$ 16,409$ for those enrolled 31-60 days). Significant reduction in use of outpatient services including chemotherapy and radiation therapy was observed among patients using hospice for at least 1 month.

Conclusions: Hospice led to cost savings by reducing utilization of aggressive care towards end-of-life among lung cancer patients. While cost savings were realized even when hospice is utilized for a short duration, large savings occurred when hospice is used for at least 1 month.

Hospice focuses on non-curative medical care, including pain and symptom management and emotional and spiritual well-being, to provide patients and their families with needed end-of-life care. ${ }^{1}$ Cancer accounts for the largest category of diagnoses leading to hospice care, representing around $27 \%$ of hospice users. ${ }^{1}$ Among the four most common cancers (breast, prostate, lung, and colorectal), hospice use is highest among lung cancer patients at around $66.7 \% .^{2}$ This is likely due to the severity of symptoms and poor prognosis of lung cancer; less than half of lung cancer patients survive beyond 1 year of diagnosis. ${ }^{3}$ 
A recent study by Kalidindi et al. found that Medicare decedents with lung cancer who used hospice had significantly lower health care spending than non-users in the last 6 months of life, with most of the difference occurring in the final month of life. ${ }^{4}$ This finding was consistent with studies of hospice use among patients with other conditions. ${ }^{5,6}$ However, one limitation of these prior studies was that that they did not distinguish between patients with different lengths of hospice enrollment; instead, they lumped together patients who used hospice for 6 months or more with those enrolled for less than a week. A second limitation of prior work was that cost savings were estimated over long time intervals - such as last year of life, ${ }^{5,6}$ last 6-months, ${ }^{4}$ or last month ${ }^{7}$, while the median length of hospice use was only 17 days, and the average length of stay was around 88.2 days. ${ }^{8}$ Given the variation in length of hospice enrollments, useful assessments of hospice savings must: 1) consider the patient's actual length of hospice stay, and 2) use the period during which hospice is actually used for cost comparisons.

A few studies examined the impact of length of hospice enrollment on spending but produced mixed results. Some found that beneficiaries enrolled in hospice longer saved Medicare dollars more. ${ }^{9,10}$ For example, Taylor et al. ${ }^{9}$ used Medicare claims data and found that hospice enrollment of 53-105 days before death maximized Medicare savings. Others showed that longer hospice stays were associated with higher Medicare spending because the costs of providing hospice exceeded savings from reduced utilization of other resources. 11,12 Kelley et al. used survey data from the Health and Retirement Study along with Medicare claims data, and found that cost savings were higher for the most common enrollment periods: $1-7,8-14$, and $15-30$ days before death, than the $53-105$ period. ${ }^{12}$ The difference in findings between these studies could be attributed to differences in study data, study periods, study samples, and study methodology. ${ }^{9,12}$ For example, Kelley et al. ${ }^{12}$ had more comprehensive data that included information on patient functional status. Thus, they were able to control for some covariates that was unavailable to Taylor et al. ${ }^{9}$

The studies by Kelley et al. ${ }^{12}$ and Taylor et al. ${ }^{9}$ included all Medicare decedents ${ }^{9,12}$ (or all cancer decedents), ${ }^{9}$ in their study sample. It is possible that the savings vary based on cancer type. For example, it is possible that for conditions such as lung cancer, longer hospice enrollments might lead to greater reductions in health spending. One study looked at the impact of hospice enrollment period on health care utilization and costs for patients with poor-prognosis cancers. ${ }^{13}$ It found that the savings were maximized when patients enrolled in hospice 5-8 weeks before death. ${ }^{13}$ However, this prior study was based on a single year of data, did not focus on a specific cancer type, and did not examine how hospice enrollment might affect utilization of different outpatient services such as chemotherapy and radiation therapy visits. Chemotherapy and radiation therapy are important drivers of cancer costs. Spending on each chemotherapy session among lung cancer patients averages between $\$ 1,250$ and $\$ 1,630$ depending on site of chemotherapy. ${ }^{14}$ Lung cancer patients also receive more radiation therapy sessions than patients with other common cancers. ${ }^{15}$ If services provided in hospice effectively substitute these aggressive services, hospice care could lead to substantial savings. Thus, examination of utilization of those select outpatient services can help us to better understand the potential mechanism by which hospice leads to savings. 
The objective of our study was to examine the role of length of hospice enrollment on expenditures and utilization among lung cancer patients. We used propensity score matching techniques to mitigate the selection bias, which is a common issue when studying the impact of hospice use. First, we created propensity score matched cohorts of hospice users and nonusers across 5 different enrollment periods -1 ) those enrolled for 1-7 days, 2) those enrolled for 8-14 days, 3) those enrolled for 15-30 days, 4) those enrolled for 31-60 days, and 5) those enrolled between 61 days -6 months. Next, we examined spending and utilization differences during the target enrollment period between hospice users and nonusers. Similar to the study by Kalidindi et al., ${ }^{4}$ we examined a comprehensive set of utilization measures including hospitalizations, ED visits, and use of select outpatient services including chemotherapy and radiation therapy sessions.

\section{Methods}

\section{Data}

Our primary data were the 2009-2013 Medicare claims including: Medicare Hospice claims; Part A Medicare Provider Analysis and Review (MedPAR) files containing inpatient hospital and skilled nursing facility (SNF) claims; Part B Medicare Hospital Outpatient files containing claims for services in hospital outpatient departments (HOPDs) and Carrier files containing claims for services by non-institutional providers; and Durable Medical Equipment (DME) files containing claims submitted by DME suppliers. The 2009 data were used only to observe 6 months of utilization prior to death for those who died in 2010.

Other data files included: Medicare Master Beneficiary Summary Files (MBSF) containing demographic characteristics and disease indicators for each beneficiary; American Community Survey (ACS) data containing zip-level income, education, and unemployment rates; and Hospice Compare files that provided information regarding the number of hospice providers serving the beneficiary's zip.

\section{Sample}

The Centers for Medicare and Medicaid Services (CMS) first identified all patients with cancer between 2009-2013 using 100\% of Medicare claims and applying the standard algorithm used for creating cancer indicators in the Medicare Chronic Condition Warehouse (CCW) files. ${ }^{16}$ The research-identifiable claims and demographic information for a $10 \%$ random sample were then provided to us. From that sample, we identified beneficiaries with an indicator for lung cancer who died between 2010 and 2013.

We included only beneficiaries that were continuously enrolled in Medicare Part A and Part B for at least 6 months before death to ensure we could observe all utilization and spending data for the sample. We removed any beneficiaries that were enrolled in Medicare Advantage (MA) because their utilization information was not available to us. These exclusions resulted in a sample of 43,594 individuals.

Patients who enrolled in hospice at any time in the 6 months before death comprised the hospice user group. Beneficiaries enrolled for longer than 6 months were excluded. All patients that did not enroll in hospice comprised the non-user group. Within the user group, 
we defined the following categories of enrollment period: those enrolled between 1-7 days, 8-14 days, 15-30 days, 31-60 days, and 61 days -6 months.

We created the matched non-user sample for each enrollment category using logistic regression. We estimated the propensity score of hospice enrollment using the covariates at the time of death. In general, the covariates included follow prior studies, ${ }^{9,13}$ and they were age, gender, race, time since diagnosis, chronic condition indicators (such as diabetes, hypertension, chronic obstructive pulmonary disease (COPD), etc.), ZIP code-level income, education, and unemployment rates, and number of hospice providers serving the ZIP code. Similar to the study by Kelley et al., ${ }^{12}$ we included a covariate for the number of hospitalizations during the 6-month period prior to the target hospice enrollment period to account for prior utilization as a predictor of subsequent utilization (further details in Appendix A1). We also added a metastasis indicator to partially capture the disease progression because the staging information was not available (Appendix A1).

Next, we matched hospice enrollees with each enrollment period to non-hospice controls who were within $2 \%$ of the standard deviation of the propensity scores. All unmatched subjects were excluded.

We assigned a hypothetical enrollment date to each non-user in a way that the time from enrollment to death was the same for the non-user as their matched user.

\section{Outcome measures}

a) Spending: For each enrollment period group, we defined the primary outcome as the total Medicare spending from the beginning of the enrollment period to death. For the nonenrolled, the enrollment date was the assigned date based on matching (described above). Spending included the Medicare allowed payments for all inpatient stays, ED visits, outpatient procedures, visits, and drugs, DME, and hospice care. All costs were adjusted for inflation to 2013 US dollars using Consumer Price Index (further details in Appendix A1).

b) Utilization: For each enrollment period group, we identified the number of hospital visits, the number of ED visits, number of Part B chemotherapy claims, and number of radiation therapy sessions from the outpatient claims data (further details in Appendix A1).

\section{Statistical Approach}

First, we descriptively compared the covariates between hospice users in each enrollment period category and the overall sample of non-users before matching. Next, using the matched groups, we compared covariates between each set of hospice users and their propensity score matched non-users to check the balance of the sample on observable covariates.

We compared unadjusted spending between hospice users and matched controls by enrollment period. We then estimated spending using generalized linear models (GLM) with log-link and gamma distribution separately for each enrollment period. ${ }^{4,12}$ For count measures of utilization, we estimated negative binomial models. ${ }^{4,12}$ The same set of covariates were included in the regression analysis as in the matching. For each outcome, we 
computed regression-adjusted predicted values among hospice users and non-users after setting all other covariates to their mean values. All standard errors were clustered by zip because patients within the same zip share similar characteristics.

\section{Sensitivity Analysis}

We also ran analysis using a separate category of enrollees enrolled for 53-105 days to compare our results with prior studies using that category. For example, Kelley et al., ${ }^{9}$ used enrollment periods of 1-7, 8-14, 15-30, and 53-105 days before death.

\section{Results}

\section{Sample Characteristics}

The characteristics of the sample before matching are summarized in Appendix Table A2. In general, patients that used hospice were likely to be older, female, white, and had a higher likelihood of metastatic cancer than non-users. Comparison of the patient characteristics in the matched samples by enrollment period is reported in Appendix Tables A3-A7. These tables indicate that matching resulted in cohorts that were better balanced on covariates.

\section{Spending measures}

Figure 1 presents comparison of unadjusted mean spending between hospice users and nonusers in matched cohort. Longer enrollment periods were associated with larger savings with the largest savings of $\$ 18,779$ realized when beneficiaries enrolled for 61 days -6 months $(\mathrm{p}<0.01)$. Figure 2 presents the regression-adjusted mean spending differences between hospice users and matched non-users by enrollment period. The trends in regressionadjusted spending by enrollment period were similar to the unadjusted measures, but the magnitude of savings was lower in the adjusted measures. Medicare lung cancer decedents that received hospice care for 61 days -6 months had spending of $\$ 20,107$ during the period enrolled compared to matched non-users who had spending of $\$ 36,762$ during the same period - a difference of $\$ 16,566(\mathrm{p}<0.01)$. The estimates of savings were $\$ 16,409(\mathrm{p}<0.01)$ for those enrolled between 31-60 days, $\$ 12,209$ ( $\mathrm{p}<0.01$ ) for those enrolled for 15-30 days, $\$ 8,678(\mathrm{p}<0.01)$ for those enrolled $8-14$ days, and $\$ 3,714(\mathrm{p}<0.01)$ for those enrolled $1-7$ days.

\section{Utilization measures}

Figure 3 presents regression-adjusted mean utilization measures for each group. In general, the utilization trends for hospitalization visits, and ED visits were similar (Panels A and B). Beneficiaries that used hospice for 61 days -6 months had significantly fewer hospital visits ( 1.02 fewer visits, $p<0.01$ ), and fewer ED visits ( 0.33 fewer visits, $p<0.01$ ), than matched non-users. The estimated differences in number of hospitalization visits were $-0.74(\mathrm{p}<0.01)$ for those enrolled between 31-60 days, -0.50 ( $\mathrm{p}<0.01$ ) for those enrolled for 15-30 days, and $-0.28(\mathrm{p}<0.01)$ for those enrolled $8-14$ days, and $-0.04(\mathrm{p}<0.01)$ for those enrolled $1-7$ days. The estimated differences in number of ED visits were $-0.21(\mathrm{p}<0.01)$ for those enrolled between $31-60$ days, $-0.14(\mathrm{p}<0.01)$ for those enrolled for $15-30$ days, -0.09 $(\mathrm{p}<0.01)$ for those enrolled $8-14$ days, and $-0.06(\mathrm{p}<0.01)$ for those enrolled $1-7$ days. 
For outpatient visits (Panels $\mathrm{C}$ and $\mathrm{D}$ ), beneficiaries that received hospice care for longer durations (e.g., 61 days -6 months) had a much lower number of chemotherapy visits $(-1.33$ visit difference, $\mathrm{p}<0.01)$, and a lower number of radiation therapy sessions $(-1.40$ visit difference, $\mathrm{p}<0.01)$ than matched non-users. Lower chemotherapy $(-0.78$ visits difference, $\mathrm{p}<0.01)$ and radiation therapy $(-0.71$ visits difference, $\mathrm{p}=0.02)$ utilization was also seen for those enrolled between 31-60 days. However, unlike hospitalizations and ED visits, lower utilization was not seen for these services across all enrollment periods. While chemotherapy use was slightly lower among those enrolled in hospice for 15-30 days before death $(-0.25$ visit difference, $\mathrm{p}=0.02)$, no significant difference in chemotherapy utilization was seen for those that were enrolled for 8-14 days or 1-7 days. For radiation therapy sessions, no difference was seen between hospice users enrolled 15-30 days and matched non-users. Conversely, for those enrolled for shorter periods of 0-7 and 8-14 days, radiation therapy sessions were greater among hospice users than non-users ( 0.64 visit difference, $\mathrm{p}=0.02$ for enrollment period $8-15$ days; 0.50 visit difference, $\mathrm{p}=0.01$ for enrollment period 1-7 days).

\section{Sensitivity Analysis}

Using a separate category of beneficiaries that enrolled for 53-105 days did not impact our results (Appendix Table A8). Savings using this enrollment category were around $\$ 16,590$ $(p<0.01)$, similar to the estimated savings for hospice enrollment of 61 days -6 months and 31 days -60 days.

\section{Discussion}

We found that longer lengths of hospice were associated with greater savings in Medicare costs among lung cancer decedents. We observed a clear gradient by length of hospice enrollment with those enrolled for longer periods of time experiencing greater reductions in spending. The savings increased substantially by each enrollment period category up to 3160 days before death, but the change beyond this category was minimal. The incremental savings from having 61 days -6 months of hospice enrollment versus 31-60 days of enrollment was only around $\$ 250$ (\$16,656 versus $\$ 16,409$ ). Our study confirms findings from prior studies that hospice saves money across different enrollment periods (up to 105 days shown by Kelley et al. ${ }^{12}$, and 233 days shown by Taylor et al. ${ }^{9}$ ). However, while the study by Kelley et al. ${ }^{12}$ found that the savings are maximized between 15-30 days, we found the savings were larger for longer enrollment periods up to 6 months. Our estimates of savings were also larger compared to the prior studies that included all Medicare patients. 9,12 Our results were likely driven by our selection of lung cancer patients, who have particularly high utilization of services and spending compared to other cancer patients in the last year of life. ${ }^{17}$

Trends in utilization clearly drove the differences in savings across enrollment periods. Hospice use reduced hospitalizations and ED visits across all enrollment periods. The number of hospitalizations decreased by 1.02 for those enrolled for 60 days to 6 months and by 0.04 for those enrolled 1-7 days before death. The adjusted decrease in number of ED 
visits was 0.34 for those enrolled for 60 days to 6 months and 0.06 for those enrolled 1-7 days before death. These findings are consistent with other studies. ${ }^{12}$

An important contribution of our study is that we show trends in utilization of outpatient services such as chemotherapy and radiation therapy differed significantly between hospice users and non-users based on the enrollment period. For example, while beneficiaries that initiated hospice only 1-7 days before death had higher utilization of services such as radiation therapy, and similar utilization of chemotherapy as their matched non-users, those that used hospice for longer than 30 days had reduced utilization of both services. Chemotherapy and radiation therapy are sometimes used for palliative purposes during endof-life. But the benefits were found to be limited considering the toxicity due to chemotherapy, the healthcare costs, and the time spent on these sessions at the hospital. ${ }^{18-20}$ Thus, reductions in aggressive care towards end-of-life could be beneficial to the patient.

\section{Limitations}

There are several limitations of this study. First, while our propensity score approach was able to match hospice users and non-users on observable characteristics, there may still be differences in unobservable characteristics. For example, we do not have information on patient preferences available to some researchers. ${ }^{12}$ Second, we did not have home health data; however, our estimates excluding home health costs are likely to be conservative because non-users could potentially use home health to substitute for services provided in hospice. Third, we did not have cancer stage information. While we used an indicator for metastasis, there may be important differences in stage that we are unable to capture. ${ }^{21,22}$ Last, enrollment period could also be impacted by time of diagnosis. A large number of lung cancer patients are diagnosed in advanced stages of the disease. ${ }^{23}$ It is possible that the difference between an individual who enrolled for 30 days versus 60 days was influenced by their time of diagnosis rather than patient preference.

\section{Conclusion}

Among lung cancer patients, hospice leads to lower spending across all enrollment periods up to 6 months before death - the current period used to determine hospice eligibility. Longer enrollment appears to be associated with greater savings, likely driven by greater reductions in utilization of different services including inpatient hospitalizations, ED visits, and chemotherapy and radiation therapy sessions. Length of hospice enrollment may be driven by a variety of factors such as patient and family choice, and provider's ability to accurately assess prognosis. Thus, it is important to assess and overcome some of the patient barriers towards hospice enrollment. Additionally, providers must weigh all patient and family factors against the benefits of early enrollment while formulating an end-of-life treatment plan.

\section{Funding source:}

NIH/NIA grant number 1R01AG047934-01, and NIH grant number R24 HD041025 


\section{Appendix A1.: Description of Methods \\ Construction of prior hospitalization variable:}

In order to ensure that we are able to construct this variable, only beneficiaries that had Part A enrollment for the period of full 12 months before death were included in our sample. The number of prior hospitalizations variable was constructed before propensity score matching and was used as one of the covariates in matching. Since it is not possible to assign an enrollment date prior to matching, we used the following approach to create the prior hospitalization variables. For all beneficiaries that utilized hospice for $1-7$ days, we calculated the number of hospitalizations in the 6-month period prior to 7 days (considering 7 days as the target enrollment period). Similarly, for those that were enrolled in hospice for 8-14 days, we constructed number of hospitalizations in the 6-month period prior to the 14 days and so on. For all non-users, we created 5 separate hospital utilization variables utilization in the 6-month period prior to 7 days before death, prior to 14 days before death, 30 days before death, 60 days before death, and 6 months before death. While matching, the prior utilization variables were selected based on the enrollment period. For example, while matching the hospice-user group that utilized hospice for 1-7 days to non-users, the variable constructed for the 6-month period prior to 7 days before death was used.

\section{Construction of metastasis variable:}

An indicator of metastasis = ' 1 ' was assigned to beneficiaries who had at least two diagnosis codes indicating metastatic disease (International Classification of Diseases, Ninth Revision, Clinical Modification (ICD-9-CM) 196-199), separated by 30 days or more. ${ }^{1}$

\section{Cost inflation adjustment}

Costs for inpatient stays and ED visits were inflated using the hospital and related services component of the consumer price index (CPI). Costs for outpatient visits, physician bills, SNF visits, and hospice care were inflated using the medical care services component of the CPI. Costs for DME will be inflated using the medical care commodities component of the CPI, and prescription drugs will be inflated using CPI for prescription drugs. These inflation methods have been used in prior studies. ${ }^{2}$

\section{Procedure codes used for constructing chemotherapy and radiation therapy visits}

Chemotherapy Drugs (J-Codes) - J9000-J9999, J8521, J8560, J8520, and J8530

Radiation therapy sessions $-77261,77262,77263,77280,77285,77290,77295,77299$, 77300, 77301, 77305, 77306, 77307, 77310, 77315, 77321, 77326, 77327, 77328, 77331, 77332, 77333, 77334, 77336, 77338, 77370, 77371, 77372, 77373, 77399, 77401, 77402, 77403, 77404, 77405, 77406, 77407, 77408, 77409, 77410, 77411, 77412, 77413, 77414, 77415, 77416, 77417, 77418, 77421, 77422, 77423, 77427, 77430, 77431, 77432, 77435, 77469, 77470, 77499, 77520, 77522, 77523, 77525, 77750, 77761, 77762, 77763, 77776, 77777, 77778, 77781, 77782, 77783, 77784, 77785, 77786, 77787, 77789, 77790, 77799 
Table A2.

Descriptive comparison of sample characteristics in full sample (before matching)

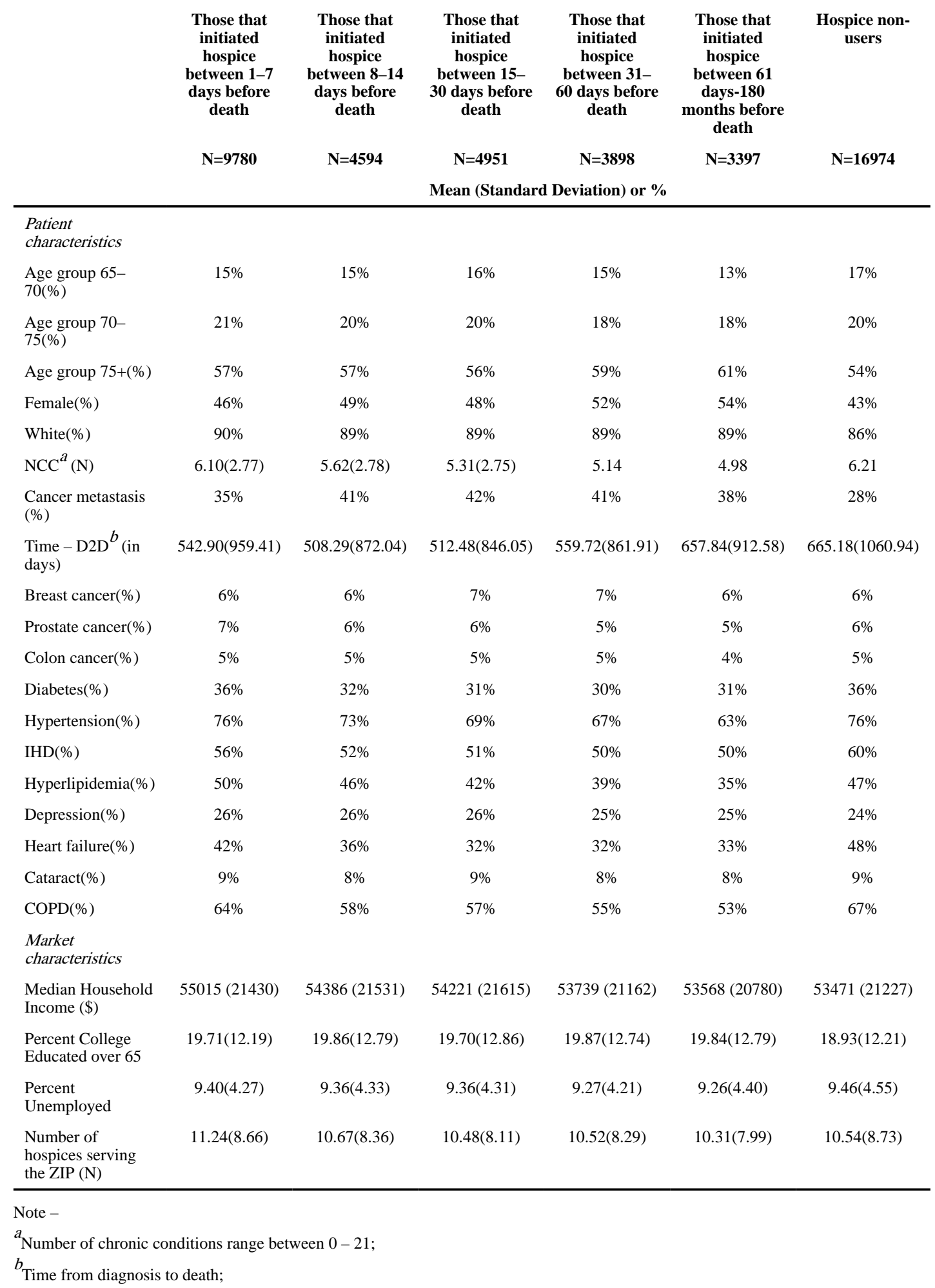

Am J Hosp Palliat Care. Author manuscript; available in PMC 2021 November 01. 
${ }^{c}$ Chronic Obstructive Pulmonary Disease;

\section{Table A3.}

Descriptive comparison of sample characteristics in matched sample ${ }^{a}$ for enrollment period category $-1-7$ days before death

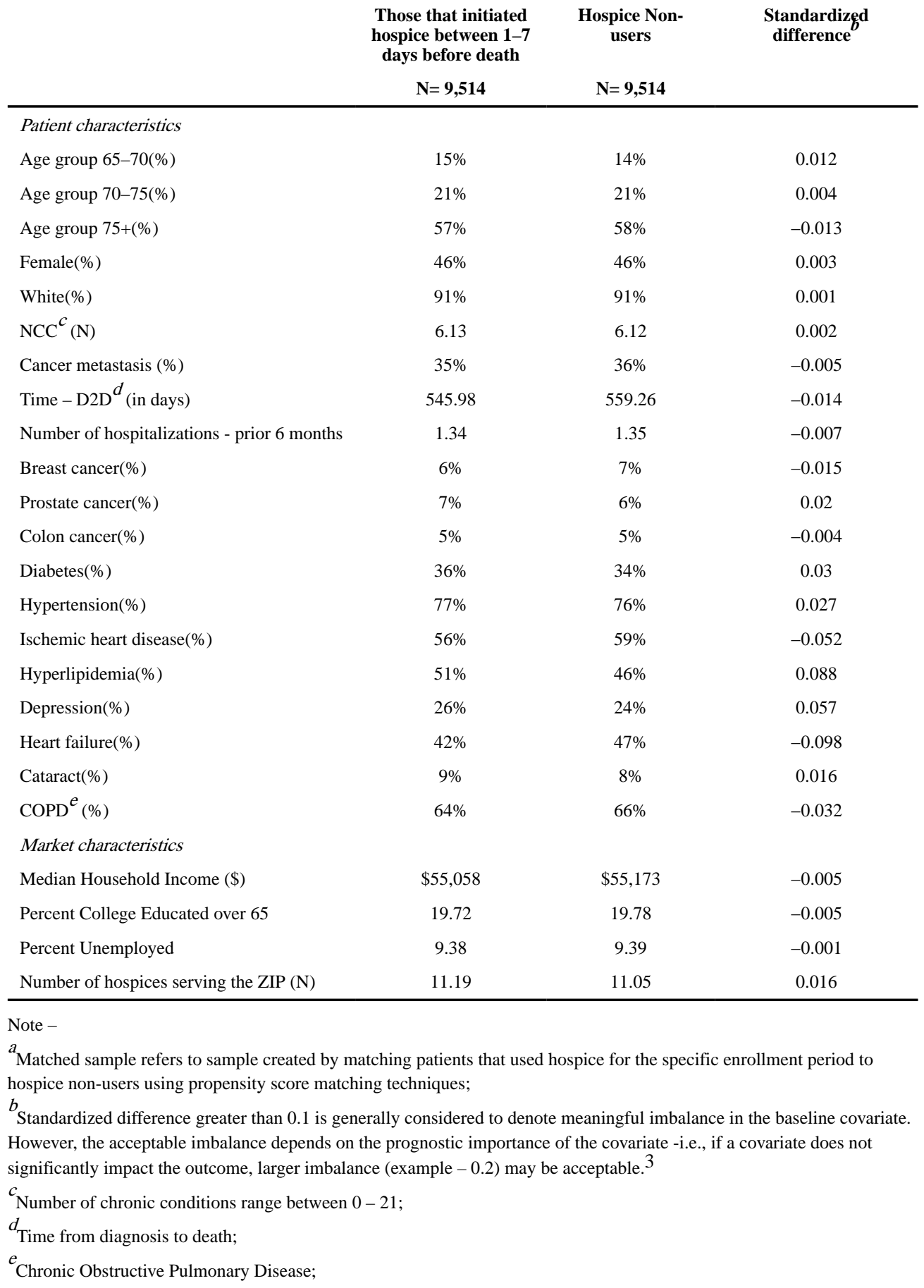

Am J Hosp Palliat Care. Author manuscript; available in PMC 2021 November 01. 


\section{Table A4.}

Descriptive comparison of sample characteristics in matched sample ${ }^{a}$ for enrollment period category $-8-14$ days before death

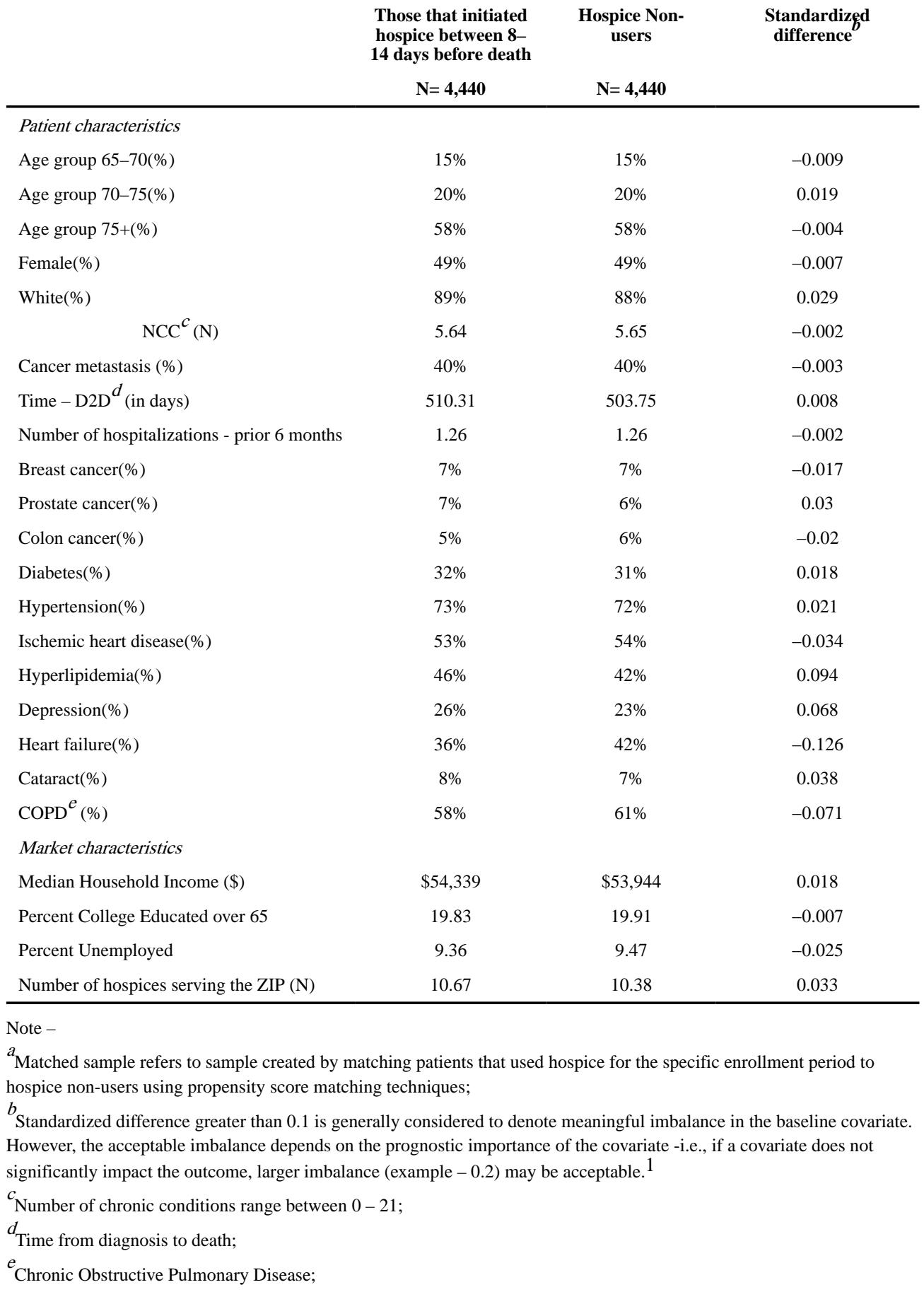

Am J Hosp Palliat Care. Author manuscript; available in PMC 2021 November 01. 
Table A5.

Descriptive comparison of sample characteristics in matched sample ${ }^{a}$ for enrollment period category - 15-30 days before death

\begin{tabular}{|c|c|c|c|}
\hline & $\begin{array}{l}\text { Those that initiated } \\
\text { hospice between 15- } \\
30 \text { days before death }\end{array}$ & $\begin{array}{l}\text { Hospice Non- } \\
\text { users }\end{array}$ & $\begin{array}{l}\text { Standardized } \\
\text { difference }\end{array}$ \\
\hline & $\mathrm{N}=\mathbf{4 , 7 8 0}$ & $\mathrm{N}=\mathbf{4 , 7 8 0}$ & \\
\hline \multicolumn{4}{|l|}{ Patient characteristics } \\
\hline Age group $65-70(\%)$ & $16 \%$ & $16 \%$ & 0.009 \\
\hline Age group $70-75(\%)$ & $20 \%$ & $20 \%$ & 0.002 \\
\hline Age group $75+(\%)$ & $56 \%$ & $57 \%$ & -0.007 \\
\hline Female(\%) & $48 \%$ & $49 \%$ & -0.012 \\
\hline White(\%) & $89 \%$ & $88 \%$ & 0.03 \\
\hline $\operatorname{NCC}^{c}(\mathrm{~N})$ & 5.36 & 5.33 & 0.011 \\
\hline Cancer metastasis (\%) & $42 \%$ & $41 \%$ & 0.011 \\
\hline Time $-\mathrm{D} 2 \mathrm{D}^{d}$ (in days) & 517.46 & 507.69 & 0.012 \\
\hline Number of hospitalizations - prior 6 months & 1.12 & 1.11 & 0.009 \\
\hline Breast cancer $(\%)$ & $7 \%$ & $7 \%$ & -0.02 \\
\hline Prostate cancer $(\%)$ & $6 \%$ & $6 \%$ & 0.018 \\
\hline Colon cancer $(\%)$ & $5 \%$ & $5 \%$ & -0.002 \\
\hline Diabetes $(\%)$ & $31 \%$ & $29 \%$ & 0.043 \\
\hline Hypertension $(\%)$ & $69 \%$ & $69 \%$ & 0.003 \\
\hline Ischemic heart disease(\%) & $52 \%$ & $51 \%$ & 0.001 \\
\hline Hyperlipidemia(\%) & $42 \%$ & $39 \%$ & 0.069 \\
\hline Depression $(\%)$ & $26 \%$ & $20 \%$ & 0.136 \\
\hline Heart failure $(\%)$ & $33 \%$ & $39 \%$ & -0.123 \\
\hline Cataract $(\%)$ & $9 \%$ & $7 \%$ & 0.078 \\
\hline $\operatorname{COPD}^{e}(\%)$ & $57 \%$ & $59 \%$ & -0.048 \\
\hline \multicolumn{4}{|l|}{ Market characteristics } \\
\hline Median Household Income (\$) & $\$ 54,315$ & $\$ 54,266$ & 0.002 \\
\hline Percent College Educated over 65 & 19.74 & 19.68 & 0.005 \\
\hline Percent Unemployed & 9.36 & 9.36 & 0 \\
\hline Number of hospices serving the ZIP $(\mathrm{N})$ & 10.51 & 10.42 & 0.01 \\
\hline \multicolumn{4}{|c|}{$\begin{array}{l}\text { a Matched sample refers to sample created by matching patients that used hospice for the specific enrollment period to } \\
\text { hospice non-users using propensity score matching techniques; } \\
b \text { Standardized difference greater than } 0.1 \text { is generally considered to denote meaningful imbalance in the baseline covariate. } \\
\text { However, the acceptable imbalance depends on the prognostic importance of the covariate -i.e., if a covariate does not } \\
\text { significantly impact the outcome, larger imbalance (example - 0.2) may be acceptable. }{ }^{1}\end{array}$} \\
\hline \multicolumn{4}{|c|}{$\begin{array}{l}{ }^{c} \text { Number of chronic conditions range between } 0-21 ; \\
d \text { Time from diagnosis to death; }\end{array}$} \\
\hline
\end{tabular}

Am J Hosp Palliat Care. Author manuscript; available in PMC 2021 November 01. 
Table A6.

Descriptive comparison of sample characteristics in matched sample ${ }^{a}$ for enrollment period category - 31-60 days before death

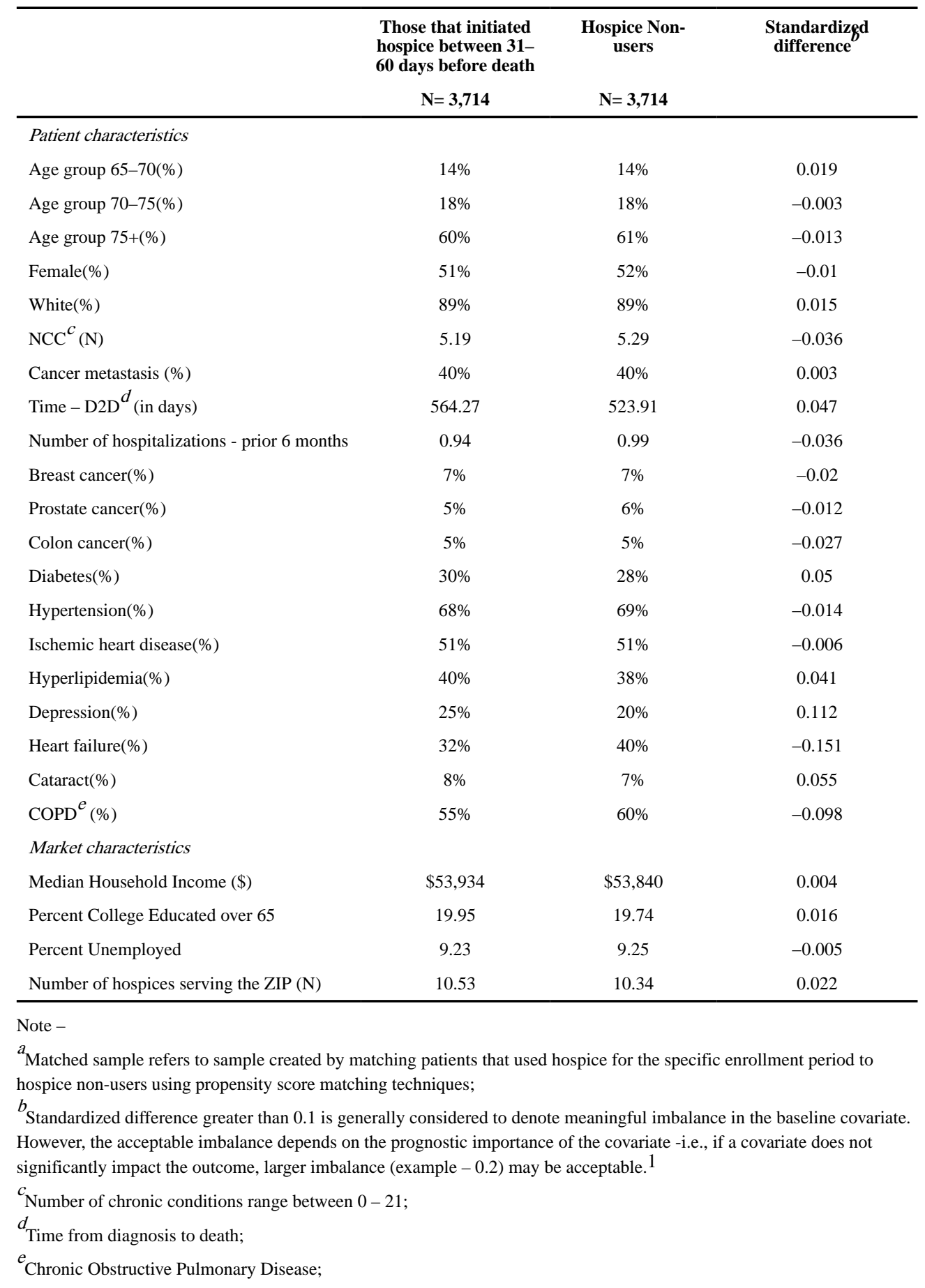

Am J Hosp Palliat Care. Author manuscript; available in PMC 2021 November 01. 


\section{Table A7.}

Descriptive comparison of sample characteristics in matched sample ${ }^{a}$ for enrollment period category -61 days- 6 months before death

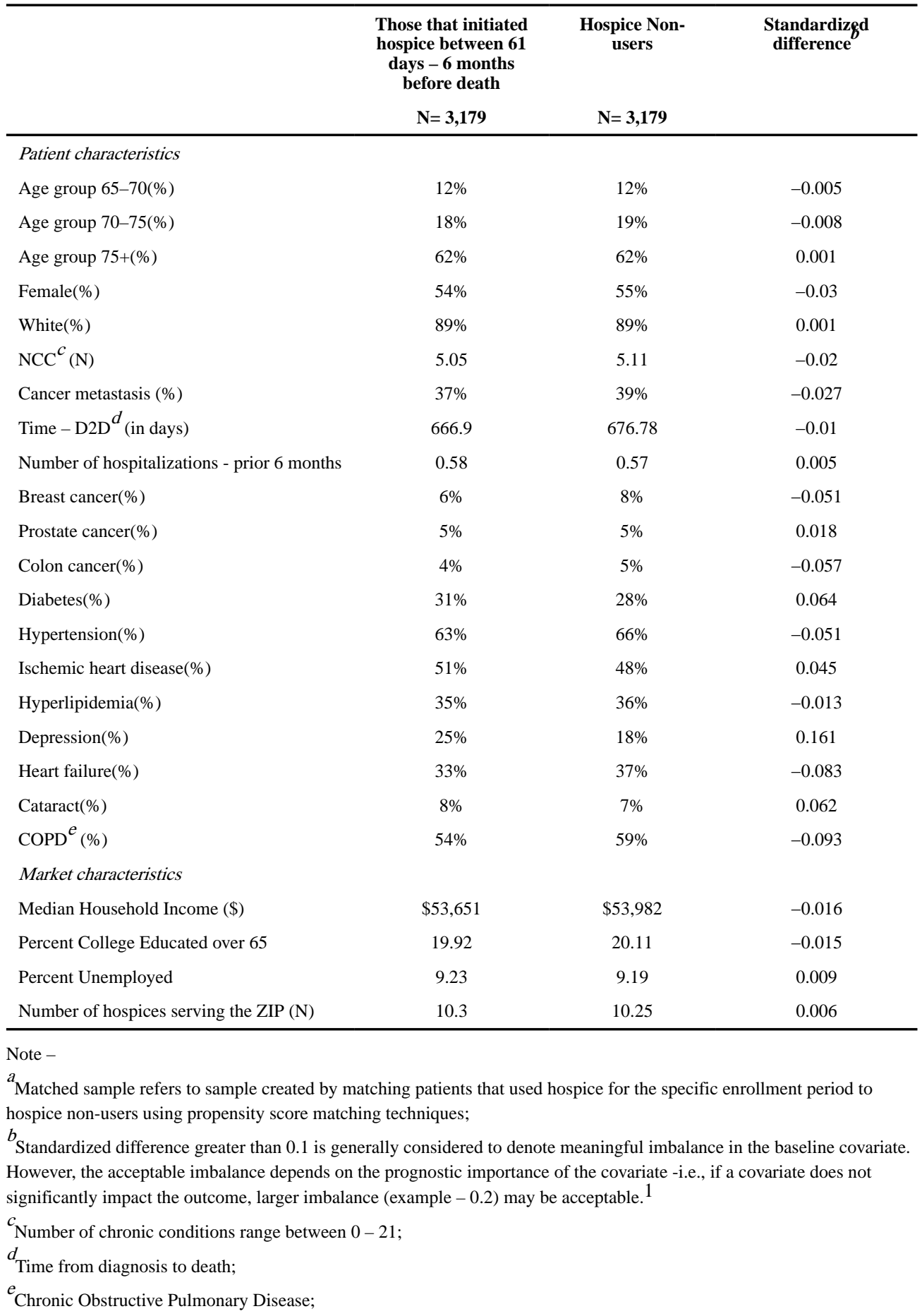

Am J Hosp Palliat Care. Author manuscript; available in PMC 2021 November 01. 


\section{Table A8.}

Sensitivity analysis - comparison of adjusted ${ }^{a}$ mean spending between hospice users and non-users using the same categories of enrollment period as prior studies

\begin{tabular}{|c|c|c|c|c|c|}
\hline & \multicolumn{4}{|c|}{ Adjusted $^{a}$ mean spending } & \multirow[b]{2}{*}{ Savings } \\
\hline & $\mathbf{N}$ & Hospice users & Non-users & P-value & \\
\hline \multicolumn{6}{|l|}{ Enrollment period category } \\
\hline $\begin{array}{l}\text { Those that initiated hospice between 1-7 days before } \\
\text { death }\end{array}$ & 19028 & 4043.346 & 7757.394 & 0 & 3714.048 \\
\hline $\begin{array}{l}\text { Those that initiated hospice between } 8-14 \text { days before } \\
\text { death }\end{array}$ & 8880 & 5080.463 & 13758.74 & 0 & 8678.277 \\
\hline $\begin{array}{l}\text { Those that initiated hospice between } 15-30 \text { days } \\
\text { before death }\end{array}$ & 9560 & 6719.86 & 18928.97 & 0 & 12209.11 \\
\hline $\begin{array}{l}\text { Those that initiated hospice between 53-105 days } \\
\text { before death }\end{array}$ & 5670 & 15826.27 & 32416.13 & 0 & 16589.86 \\
\hline
\end{tabular}

\section{References:}

1. Rao S, Kubisiak J, Gilden D. Cost of illness associated with metastatic breast cancer. Breast Cancer Res Treat. 2004;83(1):25-32.

2. Enomoto LM, Schaefer EW, Goldenberg D, Mackley H, Koch WM, Hollenbeak CS. The Cost of Hospice Services in Terminally Ill Patients With Head and Neck Cancer. JAMA Otolaryngology-Head \& Neck Surgery. 2015;141:1066-1074.

3. Austin PC. Balance diagnostics for comparing the distribution of baseline covariates between treatment groups in propensity-score matched samples. Stat Med.

2009;28(25):3083-3107. doi:10.1002/sim.3697

\section{References:}

1). Odejide OO. A policy prescription for hospice care. JAMA. 2016;315:257-258. [PubMed: 26784768]

2). National Hospice and Palliative Care Organization. Hospice patients with cancer diagnosis below 30 percent new report reveals. Retrieved at https://www.globenewswire.com/news-release/ 2018/03/28/1454878/0/en/Hospice-Patients-with-Cancer-Diagnosis-Below-30-Percent-NewReport-Reveals.html. 20183.

3). Noone AM, Howlader N, Krapcho M, Miller D, Brest A, Yu M, Ruhl J, Tatalovich Z, Mariotto A, Lewis DR, Chen HS. SEER Cancer Statistics Review, 1975-2015. Bethesda, MD: National Cancer Institute 20184.

4). Kalidindi Y, Segel J, Jung J. Impact of hospice on spending and utilization among patients with lung cancer in Medicare. American Journal of Hospice and Palliative Medicine. 2019:104990911987844. 
5). Enomoto LM, Schaefer EW, Goldenberg D, Mackley H, Koch WM, Hollenbeak CS. The cost of hospice services in terminally ill patients with head and neck cancer. JAMA OtolaryngologyHead \& Neck Surgery. 2015;141:1066-1074. [PubMed: 26447873]

6). Campbell DE, Lynn J, Louis TA, Shugarman LR. Medicare program expenditures associated with hospice use. Annals of Internal Medicine. 2004;140:269-277. [PubMed: 14970150]

7). Duggan KT, Hildebrand Duffus S, D’Agostino RB, Petty WJ, Streer NP, Stephenson RC. The impact of hospice services in the care of patients with advanced stage non-small cell lung cancer. Journal of Palliative Medicine. 2017;20:29-34. [PubMed: 27559623]

8). Medicare Payment Advisory Commission (MedPAC). March 2016 report to the Congress: Medicare Payment Policy, Chapter 3 Hospice services. http://www.medpac.gov/docs/defaultsource/reports/chapter-11-hospice-services-march-2016-report-.pdf

9). Taylor DH, Ostermann J, Van Houtven CH, Tulsky JA, Steinhauser K. What length of hospice use maximizes reduction in medical expenditures near death in the US Medicare program? Social Science \& Medicine. 2007;65:1466-1478. [PubMed: 17600605]

10). Powers BW, Makar M, Jain SH, Cutler DM, Obermeyer Z. Cost savings associated with expanded hospice use in Medicare. Journal of Palliative Medicine. 2015;18:4-401. [PubMed: 25494236]

11). Mor V, Kidder D. Cost savings in hospice: final results of the National Hospice Study. Health Services Research. 1985;20:407-422. [PubMed: 4055382]

12). Kelley AS, Deb P, Du Q, Aldridge Carlson MD, Sean Morrison R. Hospice enrollment saves money for Medicare and improves care quality across a number of different lengths-of-stay. Health Affairs. 2013;32:552-561. [PubMed: 23459735]

13). Obermeyer Z, Makar M, Abujaber S, Dominici F, Block S, Cutler DM. Association between the Medicare Hospice Benefit and health care utilization and costs for patients With poor-prognosis cancer. JAMA. 2014;312:1888-1896. [PubMed: 25387186]

14). Kalidindi Y, Jung J, Feldman R. Differences in spending on provider-administered chemotherapy by site of care in Medicare. The American Journal of Managed care. 2018;24:328-333. [PubMed: 30020752]

15). Shooshtari A, Kalidindi Y, Jung J. Cancer care spending and use by site of provider-administered chemotherapy in Medicare. American Journal of Managed Care. 2019;25:296-296. [PubMed: 31211557]

16). Chronic Conditions Data Warehouse. Medicare chronic condition charts - Chronic conditions Data Warehouse. https://www.ccwdata.org/web/guest/condition-categories.

17). Yabroff KR, Warren JL, Brown ML. Costs of cancer care in the USA: A descriptive review. Nature Clinical Practice Oncology. 2007;4:643-656.

18). von Plessen C, Aslaksen A. Improving the quality of palliative care for ambulatory patients with lung cancer. BMJ. 2005;330:1309-1313. [PubMed: 15933354]

19). Spiro SG, Rudd RM, Souhami RL, et al. Chemotherapy versus supportive care in advanced nonsmall cell lung cancer: Improved survival without detriment to quality of life. Thorax. 2004;59:828-836. [PubMed: 15454647]

20). von Plessen C, Bergman B, Andresen O, et al. Palliative chemotherapy beyond three courses conveys no survival or consistent quality-of-life benefits in advanced non-small-cell lung cancer. British Journal of Cancer. 2006;95:966-973. [PubMed: 17047644]

21). Thomas SK, Brooks SE, Daniel Mullins C, Baquet CR, Merchant S. Use of ICD-9 coding as a proxy for stage of disease in lung cancer. Pharmacoepidemiology and Drug Safety. 2002;11:709_ 713. [PubMed: 12512248]

22). Nordstrom BL, Whyte JL, Stolar M, Mercaldi C, Kallich JD. Identification of metastatic cancer in claims data. Pharmacoepidemiology and Drug Safety. 2012;21:21-28.

23). Midthun DE. Early detection of lung cancer. F1000Research. 2016;5:739. 
61 days -6 months

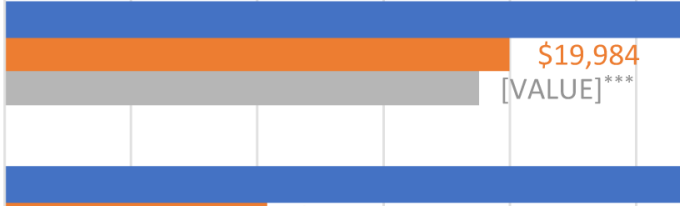

$31-60$ days

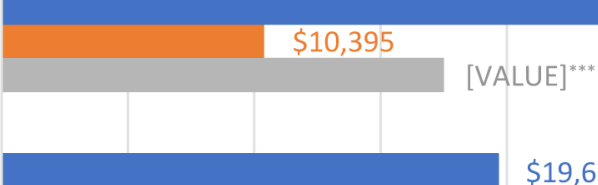

$15-30$ days

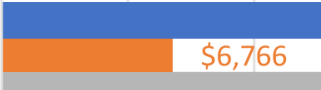

$[\text { VALUE] }]^{* * *}$

$\$ 19,636$

8 - 14 days

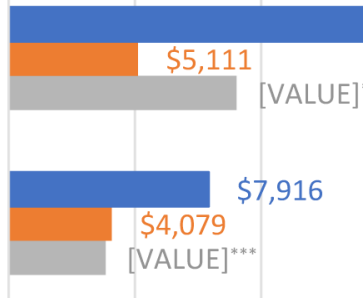

$\$ 14,133$

1-7 days

[VALUE]

$\$-$

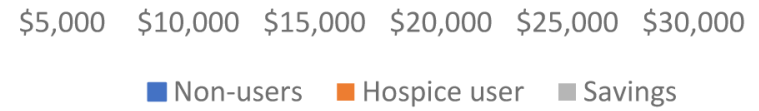

$\$ 27,910$

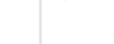

Figure 1.

Enrollment period ${ }^{a}$ category wise comparison of unadjusted mean spending between hospice-users and their matched ${ }^{b}$ non-users and estimates of savings (among Medicare beneficiaries with lung cancer)

Note: ${ }^{a}$ For hospice enrollees, enrollment period refers to the period between the hospice enrollment to death. For non-users, enrollment period refers to the periods between the assigned enrollment date (based on propensity score matched hospice user) to death. ${ }^{b}$ The matched sample of hospice users and non-users in each enrollment category. 


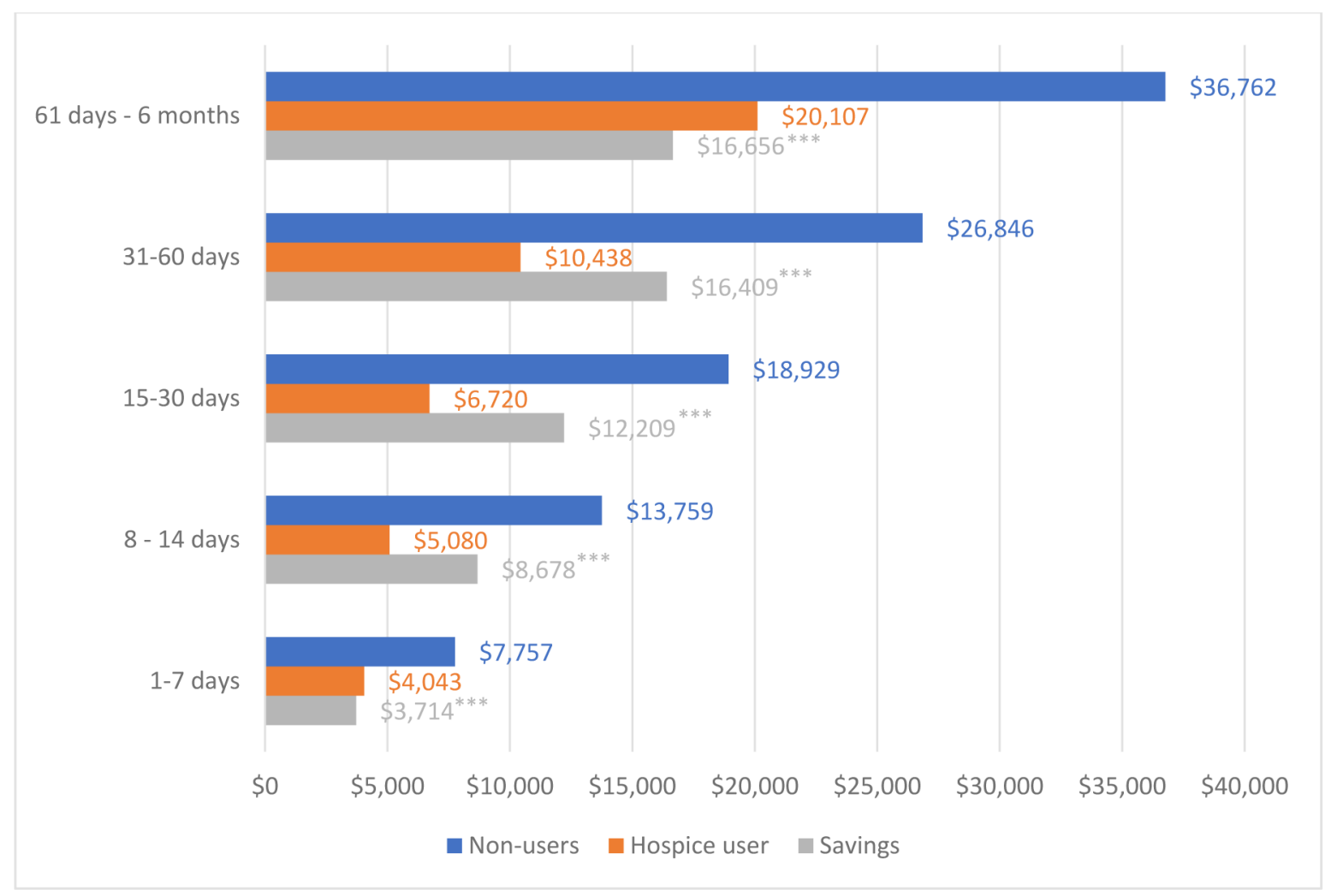

Figure 2.

Enrollment period ${ }^{\mathrm{a}}$ category wise comparison of adjusted ${ }^{\mathrm{b}}$ mean spending between hospice-users and non-users and estimates of savings (among Medicare beneficiaries with lung cancer)

Note: ${ }^{a}$ For hospice enrollees, enrollment period refers to the period between the hospice enrollment to death. For non-users, enrollment period refers to the periods between the assigned enrollment date (based on propensity score matched hospice user) to death. ${ }^{\mathrm{b}}$ Using the matched sample of hospice users and non-users in each enrollment category, spending during the enrollment period was adjusted for patient and market characteristics using GLM model with log link and gamma distribution. Standard errors accounted for clustering within a ZIP. Value are predicted values among hospice users and non-users after setting all the other covariates to their mean values; $* \mathrm{p}<0.05, * * \mathrm{p}<0.01, * * * \mathrm{p}<0.001$. 


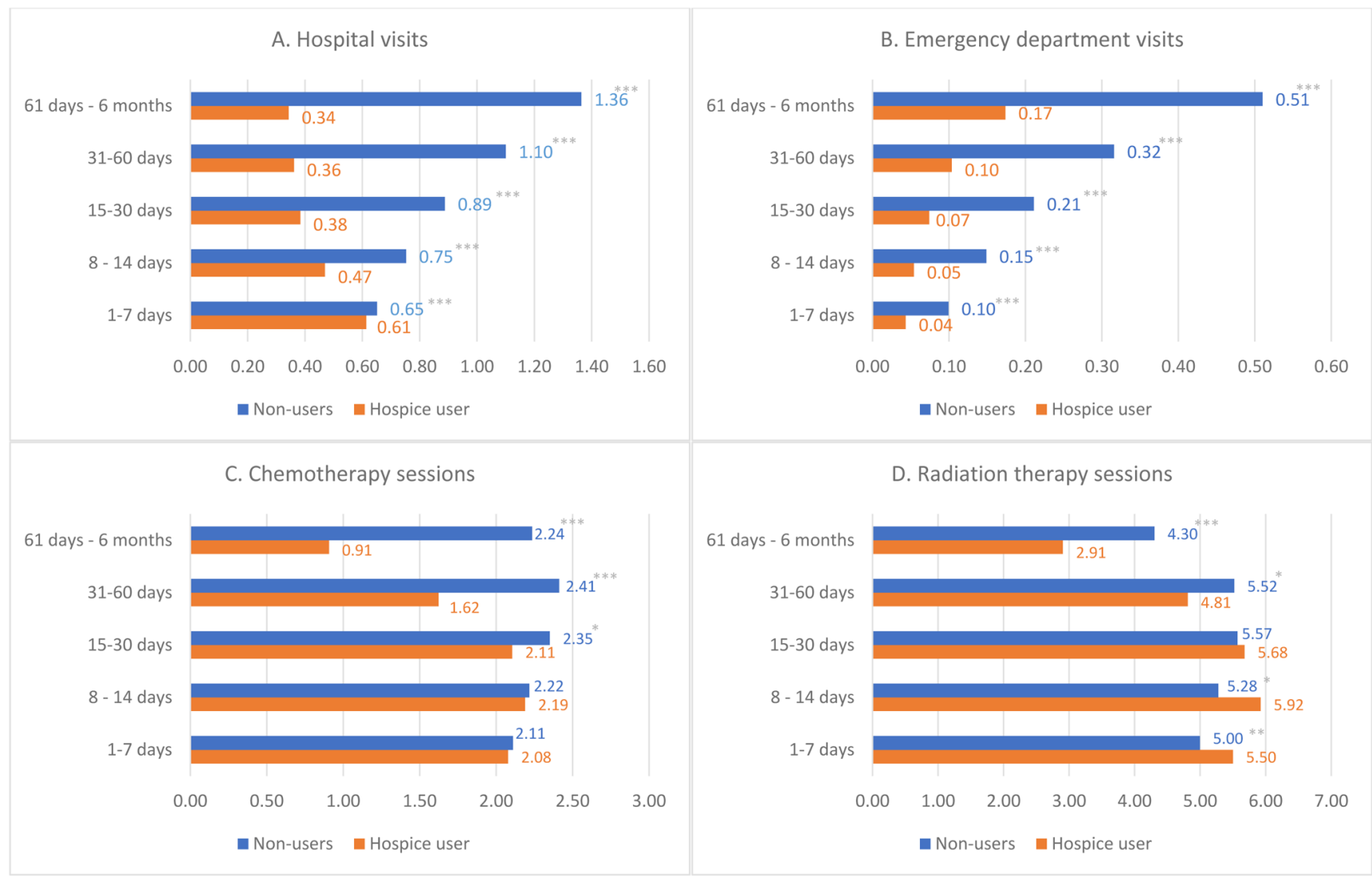

Figure 3.

Enrollment period ${ }^{\mathrm{a}}$ category wise comparison of adjusted ${ }^{\mathrm{b}}$ mean utilization between hospice-users and non-users (among Medicare beneficiaries with lung cancer)

Note: ${ }^{a}$ For hospice enrollees, enrollment period refers to the period between the hospice enrollment to death. For non-users, enrollment period refers to the periods between the assigned enrollment date (based on propensity score matched hospice user) to death. ${ }^{\mathrm{b}}$ Using the matched sample of hospice users and non-users in each enrollment category, the number of visits (counts) during the enrollment period was adjusted for patient and market characteristics using negative binomial regression models. Standard errors accounted for clustering within a ZIP. Value are predicted values among hospice users and non-users after setting all the other covariates to their mean values; *p $<0.05, * * p<0.01$, ***p $<0.001$ for the estimates of difference between the two groups. 